\title{
Kinematical Analysis of Small Biped Robot
}

\author{
Xiong Wang ${ }^{1, a^{*}}$ and Haiyan Gao ${ }^{1, b}$ \\ ${ }^{1}$ Yulin University, Yulin, Shanxi, 719000, P.R. China \\ a12296832@qq.com, b932864974@qq.com
}

Keywords: Biped Robot; Structure; Kinematics; Inverse Kinematics

\begin{abstract}
Research is carried out on the kinematics of a kind of small biped robot. The robot mechanical structure with six degrees of freedom is designed according to the bionic human body and the kinematical model of the robot is constructed through the transformation Matrix. In the inverse kinematical analysis, the relevant values are calculated. Finally, the experimental research is carried out. Results show that the robot can successfully realize walking, which proves the correctness of the kinematical analysis method in the paper.
\end{abstract}

\section{Introduction}

There are many types of robots for different purposes. In the robot family, bipedal robot has drawn the most attention with its human-like characteristic[1,2]. The structure and behaviors of biped robot are highly similar to those of humans. With the characteristics of nonlinearity, multi-variables, strong coupling and open-loop instability, it is of important theoretical significance and research value[3,4].

So far, the research on biped robot has made many meaningful achievements, but it mainly focuses on the medium and large robots. Boston Mechanics under Google developed Atias humanoid biped robot in 2013 whose size was about 1.5 times that of the adults. It had very strong balance ability and high degree of intelligence[5]. In China, Tsinghua University has developed a series of THBIP robots whose geometry sizes and mass distribution are designed with reference to the corresponding parameters of the Chinese adults with a total of 32 degrees of freedom[6]. Relative to large robot, small biped robot looks more approachable in appearance. On the premise of completing the same task, it can save resources and improve the utilization rate, so it can be used as a substitute for large biped robot in experiment. The miniaturization of biped robot must also bring many new problems to the structure design, kinematical analysis and control of robot[7,8,9,10].

This paper designs a kind of small biped robot and explores the direct and inverse kinematical modeling method. It constructs the kinematical expression through the displacement transformation matrix. At the same time, it calculates its joint angles through the inverse kinematical solution. The above method provides a feasible solution for the kinematics of biped robot.

\section{Robot Structure and Distribution of Degrees of Freedom}

The structure of the robot is shown in Fig. 1. The biped robot designed in the paper simulates the structure of human body below the waist and is simplified to be 6 degrees of freedom. The joints are driven to operate by steering gears. Each symmetric mechanical leg has three degrees of freedom, namely, hip joint, knee joint and ankle joint, respectively. 6 joints swing back and forth through controlling the mechanical connections, responsible for putting up and down the legs, so as to complete the forward and backward movement of the robot. The soles of the feet are large in size to ensure the center of gravity of the robot falls in the sole of the support leg when walking. To reduce the weight, the mechanical structures are all aluminum alloy bends in the design. 


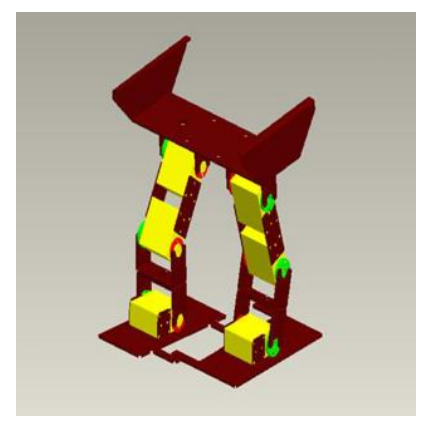

Figure 1. Robot Structure

\section{Direct Kinematical Analysis}

The legs of the robot are completely symmetrical. The coordinate system is established with the Denavit-Hartenberg method. The structure after simplification is shown in Fig. 2. It is assumed that the left leg is the swing leg and the right leg is the support leg. The reference coordinate $\{0\}$ is defined and fixed on the ankle joint of the support leg. The rotation angle of the ankle joint is $\theta_{1}$. Coordinates $\{1\}$ and $\{2\}$ are fixed on the ankle joint and hip joint with the rotation angles of $\theta_{2}$ and $\theta_{3}$, respectively. Two hip joints are solidly connected. Coordinates $\{3\}$ and $\{4\}$ are defined on the hip joint and ankle joint of the swing leg with the rotation angles of $\theta_{4}$ and $\theta_{5}$. Coordinate $\{5\}$ is defined on the ankle joint of the swing leg with the rotation angle of $\theta_{6}$.

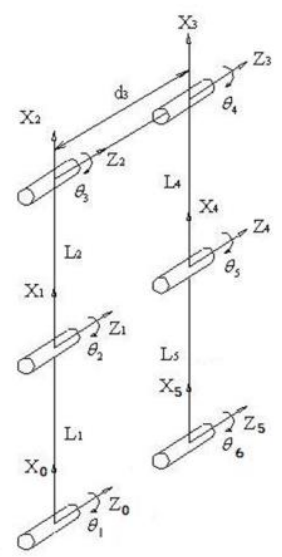

Figure 2. Structure Coordinate

$i$ is the member bar number; $\theta_{i}$ is the joint variable; $\alpha_{i}$ is the torsion angle of the connecting rod; $a_{i}$ is the length of the connecting rod; $d_{i}$ is the offset. All joint axes are parallel to each other, so the parameters of A matrix are shown in Table 1:

Table 1 Parameters of A Matrix

\begin{tabular}{|c|c|c|c|c|c|c|c|c|c|}
\hline$i$ & $\theta_{i}$ & $\alpha_{i}$ & $a_{i}$ & $d_{i}$ & $i$ & $\theta_{i}$ & $\alpha_{i}$ & $a_{i}$ & $d_{i}$ \\
\hline 1 & $\theta_{1}$ & $0^{\circ}$ & $L_{1}$ & 0 & 4 & $\theta_{4}$ & $0^{\circ}$ & $L_{4}$ & 0 \\
\hline 2 & $\theta_{2}$ & $0^{\circ}$ & $L_{2}$ & 0 & 5 & $\theta_{5}$ & $0^{\circ}$ & $L_{5}$ & 0 \\
\hline 3 & $\theta_{3}$ & $0^{\circ}$ & 0 & $d_{3}$ & 6 & $\theta_{6}$ & $0^{\circ}$ & 0 & 0 \\
\hline
\end{tabular}

According to Table 1, the homogeneous transformation matrix can be obtained as below: 


$$
\begin{aligned}
& A_{1}=\left[\begin{array}{cccc}
c_{1} & -s_{1} & 0 & L_{1} c_{1} \\
s_{1} & c_{1} & 0 & L_{1} s_{1} \\
0 & 0 & 1 & 0 \\
0 & 0 & 0 & 1
\end{array}\right] \quad A_{2}=\left[\begin{array}{cccc}
c_{2} & -s_{2} & 0 & L_{2} c_{2} \\
s_{2} & c_{2} & 0 & L_{2} s_{2} \\
0 & 0 & 1 & 0 \\
0 & 0 & 0 & 1
\end{array}\right] \quad A_{3}=\left[\begin{array}{cccc}
c_{3} & -s_{3} & 0 & 0 \\
s_{3} & c_{3} & 0 & 0 \\
0 & 0 & 1 & d_{3} \\
0 & 0 & 0 & 1
\end{array}\right] \\
& A_{4}=\left[\begin{array}{cccc}
c_{4} & -s_{4} & 0 & L_{4} c_{4} \\
s_{4} & c_{4} & 0 & L_{4} s_{4} \\
0 & 0 & 1 & 0 \\
0 & 0 & 0 & 1
\end{array}\right] \quad A_{5}=\left[\begin{array}{cccc}
c_{5} & -s_{5} & 0 & L_{5} c_{5} \\
s_{5} & c_{5} & 0 & L_{5} s_{5} \\
0 & 0 & 1 & 0 \\
0 & 0 & 0 & 1
\end{array}\right] \quad A_{6}=\left[\begin{array}{cccc}
c_{6} & s_{6} & 0 & 0 \\
s_{6} & -c_{6} & 0 & 0 \\
0 & 0 & 1 & 0 \\
0 & 0 & 0 & 1
\end{array}\right]
\end{aligned}
$$

The attitude matrix $T_{6}$ of the end connecting rod to the reference coordinate can be solved, as shown in Eq.1.

$$
T_{6}=A_{1} A_{2} A_{3} A_{4} A_{5} A_{6}=\left[\begin{array}{cccc}
c_{123456} & s_{123456} & 0 & L_{1} c_{1}+L_{2} c_{12}+L_{4} c_{1234}+L_{5} c_{12345} \\
s_{123456} & -c_{123456} & 0 & L_{1} s_{1}+L_{2} s_{12}+L_{4} s_{1234}+L_{5} s_{12345} \\
0 & 0 & 1 & d_{3} \\
0 & 0 & 0 & 1
\end{array}\right]
$$

where $c_{i \cdots n}=\cos \left(\theta_{i}+\cdots+\theta_{n}\right), s_{i \cdots n}=\sin \left(\theta_{i}+\cdots+\theta_{n}\right)$ and $i=1,2, \cdots, 6, \cdots, n=1,2, \cdots, 6$.

\section{Inverse Kinematical Analysis}

The pose matrix $T_{6}$ of the end executor of the robot and the key parameters are known to solve the joint variables to prepare for the gait analysis of the robot.

The end pose is set as shown in Eq.2:

$$
T_{6}=\left[\begin{array}{cccc}
n_{x} & o_{x} & a_{x} & p_{x} \\
n_{y} & o_{y} & a_{y} & p_{y} \\
n_{z} & o_{z} & a_{z} & p_{z} \\
0 & 0 & 0 & 1
\end{array}\right]
$$

The variable separation method is adopted to make the secondary separation of the variables to obtain Eq.3:

$$
\left\{\begin{array}{l}
L_{4} c_{34}+L_{5} c_{345}=p_{x} c_{12}-L_{1} c_{2}-L_{2}+p_{y} s_{12} \\
L_{4} s_{34}+L_{5} s_{345}=L_{1} s_{2}-p_{x} s_{12}+p_{y} c_{12}
\end{array}\right.
$$

Since the robot has many degrees of space freedom and the joint angles have many unknown solutions, it is assumed that $\theta_{1}$ and $\theta_{2}$ are known. Then, the results can be obtained as shown in Eq.4:

$$
\left\{\begin{array}{l}
k_{1}=p_{x} c_{12}-L_{1} c_{2}-L_{2}+p_{y} s_{12} \\
k_{2}=L_{1} s_{2}-p_{x} s_{12}+p_{y} c_{12} \\
\theta_{5}=\operatorname{arcos} \frac{k_{1}^{2}+k_{2}^{2}-L_{4}^{2}-L_{5}^{2}}{2 L_{4} L_{5}}
\end{array}\right.
$$

Then, the relationship between $\theta_{3}$ and $\theta_{4}$ can be solved, as shown in Eq.5:

$$
\theta_{3}+\theta_{4}=\arctan \frac{L_{4}+L_{5} c_{5}}{L_{5} s_{5}}-\arctan \frac{k_{1}}{ \pm \sqrt{\left(L_{4}+L_{5} c_{5}\right)^{2}+\left(L_{5} s_{5}\right)^{2}-k_{1}^{2}}}
$$

Eq. 6 can be obtained according to Eq.1 and Eq.2: 
The solvable $\theta_{6}$ is shown in Eq.7:

$$
\theta_{6}=\arccos n_{x}-\left(\theta_{1}+\theta_{2}+\theta_{3}+\theta_{4}+\theta_{5}\right)
$$

It can be seen from derivation that the joint angles constrain each other. Thus, in the case of confirming the space pose, 3 degrees of freedom need to be given.

\section{Experimental Verification}

Experimental verification is carried out on the constructed direct and inverse kinematical model and the solving method. The actual sizes of L1, L2, L4 and L5 of the robot prototype developed in the lab are all $40 \mathrm{~mm}$ and $\mathrm{d} 3$ is $50 \mathrm{~mm}$. The experimental analysis is based on the actual sizes.

The inverse kinematical verification is carried out through example. Assuming that $\theta_{1}=5^{\circ}, \theta_{2}=6^{\circ}$ and $\theta_{3}=8^{\circ}$, the end pose is set to be:

$$
T_{6}=\left[\begin{array}{cccc}
0.838671 & 0.544639 & 0 & 151.250973 \\
0.544639 & -0.838671 & 0 & 45.526697 \\
0 & 0 & 1 & 50 \\
0 & 0 & 0 & 1
\end{array}\right]
$$

After ruling out other unreasonable solutions, the following solution can be obtained:

$$
\theta_{1}=5^{\circ}, \theta_{2}=6^{\circ}, \theta_{3}=8^{\circ}, \theta_{4}=3.99^{\circ}, \theta_{5}=5.01^{\circ}, \theta_{6}=5.01^{\circ} \text {. }
$$

Operating the robot according to the solution can obtain the given end pose, thus proving the correctness of the algorithm.

The experimental verification is shown in Fig. 3. The robot steps the right leg with $1 / 2$ step length and the center of gravity falls on the sole of the left foot. Then, the robot steps the left leg with a step length and the center of gravity falls on the sole of the right foot. And so on, the robot can successfully complete the walking.
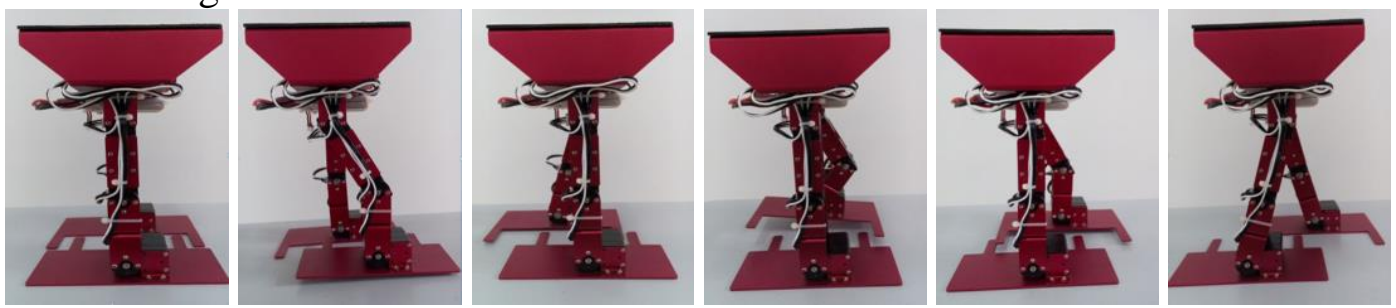

Figure 3. Experimental Verification of Foot Walking

\section{Summary}

This paper simulates the human body to design a kind of small biped robot with six degrees of freedom. Through theoretical analysis and experimental verification, it analyzes the direct and inverse kinematics of the robot, which provides the theoretical basis for the movement of biped robot. The future work will focus on further improving the movement stability of biped robot with the combination of sensor.

\section{Acknowledgements}

This work was financially supported by the university-industry cooperation projects of Yulin science and technology Bureau (2014cxy-08-02). 


\section{References}

[1] Taubes G: Science, Vol. 288 (2000) No.5463, p.80-83.

[2] Collins S, Ruina A, Tedrake R: Science, Vol. 307 (2005) No. 5712, p. 1082-1085.

[3] Fu Chenglong: Section-map stability criterion and its application for planar biped robots(Ph.D., Tsinghua University, China 2006), p.1-20.

[4] Hurmuzlu Y, Genot F and Brogliato B: Automatica, Vol. 40 (2004) No. 10, p. 1647-1664.

[5] Information on http://baike.baidu.com/.

[6] YI Qiang, CHEN Ken, LIU Li and FU Chenglong: Robot, Vol. 31 (2009) No. 6, p. 586-593.

[7] Sakagami Y, Watanabe R and Aoyama C. The intelligent ASIMO: System overview and integration[C]//IEEE/RSJ International Conference on Intelligent Robots and Systems. Piscataway,NJ, USA: IEEE, 2002: 2478-2483.

[8] Kaneko K, Kanehiro F, Kajita S, et al. Design of prototype humanoid robotics platform for $\mathrm{HRP}[\mathrm{C}] / / \mathrm{IEEE} / \mathrm{RSJ}$ International Conference on Intelligent Robots and Systems. Piscataway, NJ,USA: IEEE, 2002: 2431-2436.

[9] Geppert L and QRIO: IEEE Spectrum, Vol. 41 (2004) No. 5, p. 34-37.

[10] L"offler K, Gienger M and Pfeiffer F: International Journal of obotics Research, Vol. 22 (2003) No. 4, p. 229-239. 